\title{
PSO-HC: Particle Swarm Optimization Protocol for Hierarchical Clustering in Wireless Sensor Networks
}

\author{
Riham S. Elhabyan \\ School of Electrical Engineering and Computer Science \\ University of Ottawa, Ottawa, Ontario, Canada \\ Email: relha105@uottawa.ca
}

\author{
Mustapha C.E. Yagoub, Senior Member, IEEE \\ School of Electrical Engineering and Computer Science \\ University of Ottawa, Ottawa, Ontario, Canada \\ Email: myagoub@uottawa.ca
}

\begin{abstract}
Clustering is an efficient topology control approach for maximizing the lifetime and scalability of Wireless Sensor Networks (WSNs). Many cluster-based routing techniques have been proposed in the literature. However, in most of the proposed protocols, the communication between a sensor node and its designated cluster head $(\mathrm{CH})$ is assumed to be single-hop. Multihop communication can be used when the communication range of the sensor nodes is limited or the number of sensor nodes is very large in a network. Moreover, they used a predetermined percentage of $\mathrm{CHs}$ regardless of the network density or the number of live nodes.

Clustering is known to be non-deterministic polynomial (NP)hard problems for a WSN. Particle Swarm Optimization (PSO) is a swarm intelligent approach that can be applied for finding fast and efficient solutions of such problems. In this paper, we propose a novel centralized PSO protocol for Hierarchical Clustering (PSO-HC) in WSNs. Our objective is to maximize the network lifetime by minimizing the number of active CHs and to maximize the network scalability by using two-hop communication between the sensor nodes and their respective CHs. The effect of using a realistic network and energy consumption model in cluster-based communication for WSN was investigated. Extensive simulations show that PSO-HC outperforms the well-known cluster-based sensor network protocols in terms of average consumed energy and throughput.
\end{abstract}

Keywords-PSO, Cluster Head, WSN, RSSI, Energy Model, Hierarchical Clustering.

\section{INTRODUCTION}

Wireless Sensor Network (WSN) has emerged as a powerful technological platform with tremendous and novel applications. It has become an important technology in realizing many applications including both simple phenomena monitoring applications and heavy-duty data streaming applications such as military operations, environment monitoring and surveillance systems.

There are some factors that affect designing and operating WSN. These factors include energy efficiency and awareness, connection maintenance, minimum resource usage limitation, low latency, network coverage and load balancing in terms of energy used by sensor nodes. Due to these unique inherent characteristics it is a challenging task to select or propose a new routing or communication algorithm for a specific WSN application [1].

Using clustering techniques in WSN can help solving some of those concerns, by organizing the network nodes into smaller clusters and elect a cluster head $(\mathrm{CH})$. In clusterbased protocols, the network operating time is divided into rounds. Each round consists of two phases, the set-up phase and the steady-state phase. In the set-up phase, the network is configured. The $\mathrm{CH}$ nodes and the clusters are determined, and each $\mathrm{CH}$ assigns a member node to a slot in order to create time-division multiple-access (TDMA) schedule. In the steadystate phase, each member node sends its data to its respective $\mathrm{CH}$ at the assigned time slot and the $\mathrm{CH}$ aggregates the data and forward it to a central Base Station (BS) [2].

The election of $\mathrm{CHs}$ depends on several factors such as its residual energy and neighbour density. Since the $\mathrm{CH}$ has to stay active during the entire round, it may drain its energy earlier than the other members of the cluster. So the role of cluster head is periodically rotated among the sensor nodes to ensure balanced energy consumption. Since the $\mathrm{CHs}$ are constantly active during the entire round, minimizing the number of $\mathrm{CHs}$ will in turn reduces the energy consumption and increase the network lifetime. Therefore, the number of elected $\mathrm{CHs}$ is an important factor that affects the network lifetime significantly.

The objective of clustering is to search among a group of sensor nodes to find a set of nodes that can act as clusterheads. For a given network topology, it is difficult to find the optimal set of $\mathrm{CH}$ nodes. For $N$ sensor nodes, there are $2^{N}-1$ different combination of solutions, where in each solution, a sensor node is either elected as $\mathrm{CH}$ or non-CH [3]. This has been proved to be a Non-deterministic Polynomial (NP)-hard optimization problem [3].

Solutions to NP-hard problems involve searches through huge spaces of possible solutions. Swarm intelligence approaches have been applied successfully to a variety of NPhard problems.

Particle swarm optimization (PSO) is a swarm intelligence based optimization method. PSO has many advantages over other alternatives optimization techniques like Genetic Algorithms (GA). For example, ease of implementation on hardware or software, high-quality solutions because of its ability to escape from local optima and quick convergence [4] [5]. Because of its effectiveness in solving NP-hard problems, PSO has been adopted to optimize the $\mathrm{CH}$ election by several centralized clustering protocols. Clustering is a repeated process; therefore, the simpler the optimization algorithm, the better the network efficiency is. This is another reason PSO is a popular choice for WSN clustering.

The remainder of this paper is organized as follows. Section 2 reviews the related work on clustering protocols and the associated drawbacks. The proposed system model is presented 
in section 3. Section 4 provides a brief summary of PSO. Section 5 provides a detailed description of the proposed protocol. Simulations results will be illustrated in section 6 . Finally, we concluded our work and highlighted a few future directions in Section 7.

\section{RELATED WORK}

Clustering protocols have been studied extensively to improve the performance of WSN [6] [2]. However, all the clustering protocols proposed so far present some drawbacks.

Low energy adaptive clustering hierarchy (LEACH) [7] [8] is one of the most popular distributed cluster-based routing algorithms in WSN that has been proven to be an effective approach to prolong the network lifetime. LEACH uses a time division multiple access (TDMA) principle to avoid collisions, and in order to maintain a balanced energy consumption, suggests that each node probabilistically becomes a cluster head. However, the cluster heads are selected without considering the residual energy or the other properties of the sensor nodes. This random mechanism of selecting the cluster heads does not guarantee even distribution of clusters over the network [9].

LEACH-centralized (LEACH-C) [8] is a centralized version of LEACH. Unlike LEACH, where nodes self-configure themselves into clusters, LEACH-C uses the BS for cluster formation. Initially, each node sends its information (location and energy level) to the BS, which will use this information to find a predetermined number of cluster heads and configure the network into clusters. The clusters are chosen to minimize the energy required for non-cluster head nodes to transmit their data to their respective cluster heads. It yields better results than $\mathrm{LEACH}$ in terms of packet delivery rate and energy consumption.

Hybrid energy-efficient distributed Clustering (HEED) [10] is another distributed clustering protocol that is an extension of LEACH. Cluster formation is achieved with an iterative approach. Cluster heads selection in this protocol is primarily based on the residual energy of each node. To increase energy efficiency and further prolong network lifetime, a secondary clustering parameter considers intra-cluster "communication cost" is introduced which can be a function of neighbour proximity or cluster density. The main objectives of HEED are to distribute energy consumption to prolong network lifetime, minimize energy during the $\mathrm{CH}$ selection phase, and reduce the control overhead of the network. The improvement over LEACH is that HEED can evenly distribute the cluster heads in the sensing area by local competition.

Energy-Efficient Clustering Scheme (EECS) [11] is another distributed clustering protocol. EECS extends LEACH algorithm by dynamic sizing of clusters based on cluster distance from the BS. In the cluster head election phase, the cluster head is elected by localized competition and its no iteration property makes it differ from HEED. This competition involves candidates broadcasting their residual energy to neighbouring candidates. If a given node does not find a node with more residual energy, it becomes a cluster head. However, the EECS protocol does not consider the structural characteristics of network topology and thus cluster heads are elected on the basis of residual energy. Furthermore, because the set of candidate nodes in the competition are selected randomly before the competition, this may result in non-optimal $\mathrm{CH}$ selection.

An energy-aware clustering for WSNs using PSO algorithm (PSO-C) is a centralized clustering protocol implemented at the BS [12]. It considers both energy available to nodes and physical distances between the nodes and their CHs. This protocol defines a cost function which tries to minimize both the maximum average euclidean distance of nodes to their associated $\mathrm{CHs}$ and the ratio of total initial energy of all nodes to the total energy of the $\mathrm{CH}$ candidates. It also ensures that only nodes with sufficient energy are selected as CHs. PSO$\mathrm{C}$ outperforms both LEACH and LEACH-C in terms of the network lifetime and the throughput. Authors in [13] showed that PSO-C outperforms GA and K-means-based clustering protocols as well in terms of convergence time, network lifetime and data delivery.

All these protocols try to extend the network lifetime, using some parameters for the $\mathrm{CHs}$ selection and cluster formation. They use a predetermined number of $\mathrm{CHs}$. However, there is a trade-off between the number of $\mathrm{CHs}$ elected and the network lifetime. Since the $\mathrm{CHs}$ are constantly active during the entire round, minimizing the number of $\mathrm{CHs}$ will in turn reduces the energy consumption and increase the network lifetime. Moreover, using a predetermined number of $\mathrm{CH}$ does not guarantee to cover the whole network. However, using multi-hop communication between the sensor nodes and their respective $\mathrm{CHs}$ will create more scalable WSNs and solve the problem of the limited transmission range.

In addition to the previously mentioned problems and up to our best knowledge, all the clustering protocols that were proposed so far use the energy consumption model suggested in [8], an energy model fundamentally flawed for modelling radio power consumption in sensor networks. It ignores listening energy consumption, which is known to be the largest contributor to expended energy in WSN. Moreover, most of the link quality-based clustering protocols proposed, such as [8], [11], [12], [14], assume that each node is equipped with self-locating hardware such as a GPS. Though this is a simple solution, the resulting cost renders that solution inefficient and unrealistic [15]. Furthermore, several studies has shown that link quality in WSN is not correlated with distance [16]-[19].

Based on the above discussions, in this paper we propose a novel and realistic centralized PSO-based hierarchical clustering protocol (PSO-HC) for WSNs. The objective of the protocol is to find the optimal number of $\mathrm{CHs}$ to minimize the average energy consumption. Furthermore, the protocol tries to maximize the network coverage and scalability by constructing two-hop clusters. Moreover, we investigated using a realistic network and energy consumption model in our proposed protocol.

\section{The SySTEM MODEL}

Our proposed protocol relies on the following key realistic assumptions regarding the Wireless Sensor network:

\section{A. The WSN model}

A set of sensor nodes is randomly spread throughout a two-dimensional square field. Additionally, no assumptions are 
made about the network density. We consider the following properties of the sensor network:

- The sensor nodes are static; in the majority of applications, sensor nodes have no mobility.

- Initially all sensor nodes are charged with the same amount of energy.

- Communication links are bidirectional.

- The computation and communication capabilities are the same for all network nodes.

- The sensor nodes are unaware of their location.

\section{B. The energy consumption model}

In the proposed approach, we used a realistic energy consumption model based on the characteristics of the Chipcon CC2420 radio transceiver data sheet [20]. The total energy consumed by node $i, E_{i}$, is calculated as follows [21]:

$$
E_{i}=\sum_{\text {statej }} P_{\text {statej }} \times t_{\text {statej }}+\sum E_{\text {transitions }}
$$

The index statej refers to the energy states of the sensor: sleep, reception, or transmission. $P_{\text {statej }}$ is the power consumed in each statej, and $t_{\text {statej }}$ is the time spent in the corresponding state. Moreover, the energy spent in transitions between states, $E_{\text {transitions, }}$, is also added to the node's total energy consumption. The different values of $P_{\text {statej }}$ and $E_{\text {transitions }}$ can be found in [20].

\section{PARTICLE SWARM Optimization}

Particle Swarm Optimization (PSO) is a stochastic population based optimization algorithm that was introduced by [22] and inspired by social behaviour of bird flocking or fish schooling.

The basic PSO comprises a swarm of $S$ particles (potential solutions), which fly through a D-dimensional problem search space in search of the global optimum position that produces the best fitness of an objective function [22].

Initially, each particle $i$ is randomly assigned a position $x_{i d}$ and a velocity $v_{i d}(i=1,2, \ldots, S)$, and $d=(1,2, \ldots, D)$. In every iteration, each particle adjusts its velocity to follow two best solutions. The first is the cognitive part, where the particle follows its own best solution found so far. This is the solution that produces the lowest cost (has the highest fitness). This value is called pbest ${ }_{i}$ (particle best). The other best value is the current best solution of the swarm, i.e., the best solution by any particle in the swarm. This value is called gbest (global best).

After finding the two best values, particle $i$ then updates both its position and velocity iteratively with the following equations:

$$
\begin{aligned}
& v_{i d}(t+1)=w \times v_{i d}(t)+ \\
& c_{1} \times r_{1} \times\left(\text { pbest }_{i}(t)-x_{i d}(t)\right)+c_{2} \times r_{2} \times\left(\text { gbest }(t)-x_{i d}(t)\right) \\
& x_{i d}(t+1)=x_{i d}(t)+v_{i d}(t+1)
\end{aligned}
$$

The parameters, $c_{1}$ and $c_{2}$ are two positive constant named as learning factors, usually set as $c 1=c 2=2 . r_{1}$ and $r_{2}$ are random variables between $[0,1] . w$ is a weight factor that control the velocity of the particle.

The PSO algorithm is shown below:

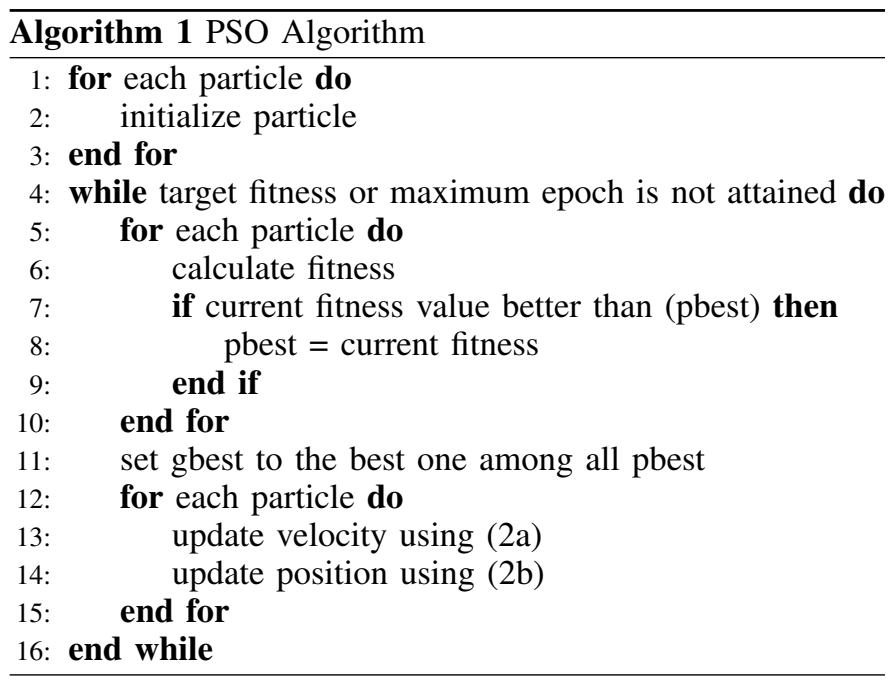

\section{The Proposed Protocol}

In this paper, we adopt a centralized and PSO-based clustering protocol to create hierarchical cluster structure for the sensor nodes.

In the proposed clustering protocol, the network operating time is divided into rounds. Each round consists of two phases, the set-up phase and the steady-state phase.

\section{A. The Set-up Phase}

The main goal of this phase is to find the optimal set of $\mathrm{CHs}$ and form the clusters.

The set-up phase starts with neighbour discovery where each sensor node in the network broadcasts a HELLO packet that includes its ID. A sensor node that receives this HELLO packet will update its neighbour table with the ID included in the packet along with the Received Signal Strength Indicator (RSSI) value in the received packet.

After the neighbour discovery ends by all the sensor nodes, the protocol uses flooding method to transfer the control data to the BS. Each node broadcast the following data about itself: ID, residual energy and it neighbour table data. A node that receives this packet will rebroadcast it till it reaches the BS.

Based on the information the BS received, the BS will computes the average energy level of all nodes. Only nodes with an energy level above the average are eligible to be a $\mathrm{CH}$ candidate for this round to ensure that only nodes with sufficient energy are selected as CHs. Next, the BS runs PSO algorithm to find the best $K \mathrm{CHs}$. A particle is represented as a sequence of candidate CHs ID's.

1) Particle Initialization: In the proposed protocol, each particle's position vector that represents the $\mathrm{CH}$ nodes IDs is initialized with random integer values in the range $[1$, networksize -1$]$ where node ID 0 represents the BS. Only nodes with an energy level above the average are eligible to 
be a $\mathrm{CH}$ candidate for this round to ensure that only nodes with sufficient energy are selected as $\mathrm{CHs}$.

The particle size is equal to the upper bound on the number of $\mathrm{CH}$ candidates. It should be noted that the velocity update by (2a) gives non-integer velocity values, which are converted to the nearest integer in the implementation. In the case that a particle generates duplicate ID's while initialization or after position update, the unique ID's generated are used as $\mathrm{CH}$ candidates.

Fig. 1 shows an example of two different particles; that has an upper bound on the number of $\mathrm{CHs}$ equals to 5 , and the $\mathrm{CH}$ candidates generated from them.

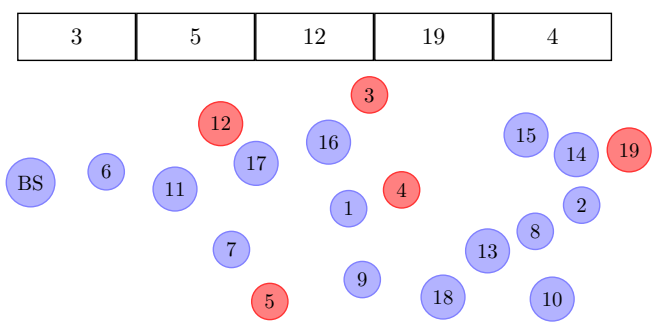

(a) A particle position vector that has no duplicates; number of $\mathrm{CHs}=5$

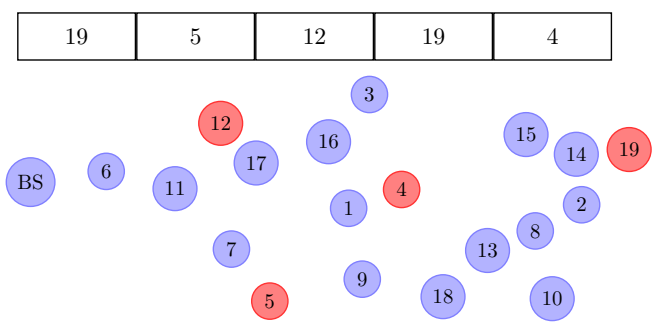

(b) A particle position vector that has duplicates; number of $\mathrm{CHs}=4$

Fig. 1. Example of two different particles and their respective $\mathrm{CH}$ candidates, upper bound $=5$, red nodes are $\mathrm{CHs}$

2) Cluster Formation: The cluster formation phase is done at the BS after generating the $\mathrm{CHs}$ from the particle. We aim at designing two-tier clusters with the objective of improving the network scalability and minimizing the number of active CHs during each round.

The BS constructs the first tier clusters by assigning each non- $\mathrm{CH}$ node to a $\mathrm{CH}$ according to the RSSI value for the link between them. In the case of multiple $\mathrm{CHs}$, the node will become a member of the $\mathrm{CH}$ having the maximum RSSI strength. Any $\mathrm{CH}$ in the first tier is called Primary $\mathrm{CH}(\mathrm{PCH})$ and has to stay active during the entire round without any sleep schedule.

The second tier is constructed by the BS by clustering all the non-CHs nodes that remained un-clustered from the first tier. The BS assigns each non- $\mathrm{CH}$ node in the second tier to a node in the first tier according to the RSSI value for the link between them. A node in the first tier that has members from the second tier is called Secondary $\mathrm{CHs}(\mathrm{SCH})$ and does not need to be active during the entire round and is set to sleep after it transmits both its own data packets and its members' data packets.

After the cluster formation ends, a node in the network can either be a $\mathrm{PCH}, \mathrm{SCH}$, a cluster member $(\mathrm{CM})$ or un-clustered node (UN). The cluster formation process is illustrated in Fig. 2 .

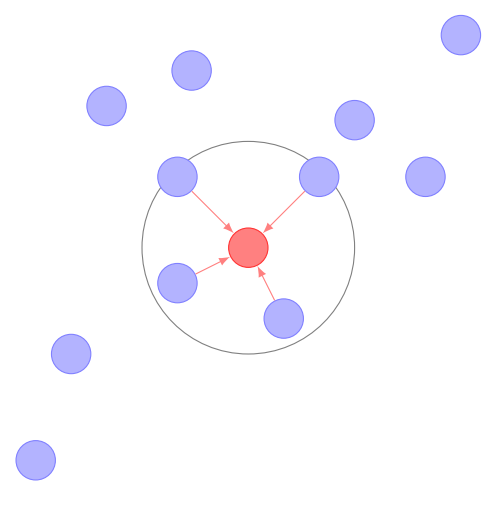

(a) Construction of the first tier

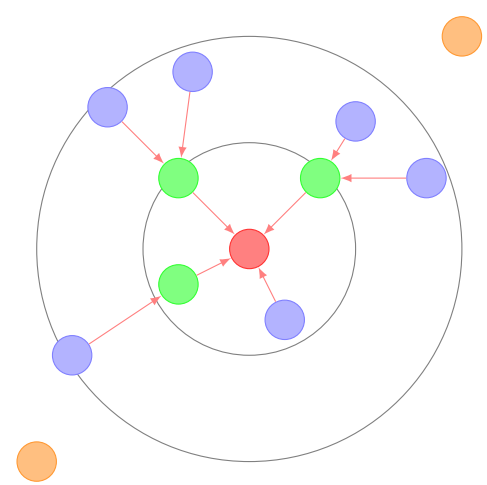

(b) Construction of the second tier

$\bigcirc \mathrm{PCH} \bigcirc \mathrm{SCH} \bigcirc \mathrm{CM} \bigcirc$

Fig. 2. Cluster Formation Process for the PSO-HC

3) Objective Function: The best $\mathrm{CH}$ are selected such that they optimize the combined effect of the following properties: energy efficiency and link quality.

1) Energy Efficiency:

To achieve an energy efficient clustering protocol, less number of $\mathrm{CHs}$ need to be active during each round. To achieve that, the protocol needs to minimize the number of $\mathrm{CHs}$. Minimizing the following function will achieve that objective:

$$
E E_{p}=\frac{K}{D}
$$

$K$ is the total number of $\mathrm{CH}$ candidates generated from particle $p$ after removing all duplicates IDs. $D$ is the upper bound on the number of $\mathrm{CHs}$. 


\section{2) Link Quality:}

The aim of this sub-objective is to maximize the link quality between the cluster members and their respective $\mathrm{CHs}$ in order to maximize the Packet Delivery Rate (PDR). RSSI, strength of the received RF signal, is one fundamental indicator of link quality. RSSI is a register in the CC2420 transceiver that is used to measure the receiver-side link quality. Several studies proved that RSSI can provide a quick and accurate estimate of whether a link is of very good quality [16]-[19].

Let $L Q_{\left(n_{i}, n^{2} x t H o p\right)}$ be an indicator of the link quality between cluster member $n_{i}$ and its next Hop (which could be $\mathrm{PCH}$ or $\mathrm{SCH})$. It can be calculated using:

$$
L Q_{\left(n_{i}, \text { nextHop }\right)}=\frac{R S S I\left(n_{i}, \text { nextHop }\right)}{\operatorname{minRSSI}}
$$

$R S S I\left(n_{i}\right.$, nextHop $)$ is the RSSI for the link from $n_{i}$ to nextHop and $\operatorname{minRSSI}$ is the worst RSSI value among all communicating pairs. The higher the value of $L Q$, the worse is the link quality.

Let $t$ refers to tier number $t$. In the proposed protocol, the maximum value of $t, T$, is 2 . If $T=1$, this means that the whole network nodes were clustered using one tier only.

To maximize the cluster quality in terms of link quality, the following sub-objective is used to minimize the sum of the worst link qualities among all the tiers:

$$
C Q_{p}=\sum_{t=1}^{T} \max _{\forall n_{i} \in \text { tier }_{t}} L Q_{\left(n_{i}, \text { nextHop }\right)}
$$

After calculating the previous sub-objectives, the final objective function that needs to be minimized is:

$$
w c_{1} \times E E_{p}+w c_{2} \times C Q_{p}
$$

$w c_{1}$ and $w c_{2}$ are weight coefficients that specify the contribution of each sub-objective in the main objective function.

4) TDMA Scheduling: The BS creates a schedule based on TDMA to allocate time slots for the cluster members according to the cluster size.

Each CM, whether it is in the first tier or the second tier, is assigned a unique TDMA turn and slot. To illustrate this, Fig.3 depicts an example of TDMA assignment on a given cluster. In this example, each node is labelled in the form Node ID/TDMA turn. Node $A$ is the first node to transmit its data, after its finishes its time slot, it goes into sleep mode to save its energy. The next node to transmit its data is $B$. Since $B$ is an $\mathrm{SCH}$, it has to wait in $R X$ (Receive) mode till node $C$ transmit its data. After $C$ transmits its own data, it goes to sleep mode then node $B$ goes to sleep mode since it has no more members. This process continues until the last node, $I$, transmit its data and go to sleep mode. The process is repeated from node $A$ again till the round ends.

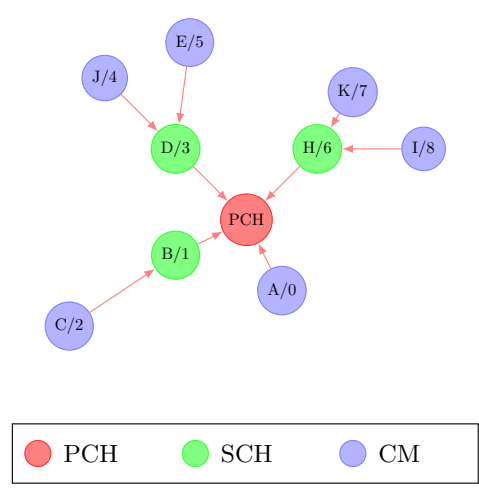

Fig. 3. TDMA Schedueling

After the BS finishes the network configuration, the BS uses flooding again to transfer the configuration to all the nodes. It broadcasts a packet containing that configuration. Each node that receives that packet will modify its status to either a $\mathrm{PCH}, \mathrm{SCH}, \mathrm{CM}$ or UN. A cluster member will update its respective $\mathrm{PCH}$ or $\mathrm{SCH}$ and TDMA schedule. A node that is not $\mathrm{CM}$ or $\mathrm{PCH}$ or $\mathrm{SCH}$ is set to sleep to save its energy.

\section{B. The Steady-up Phase}

In the steady-state phase, each member node uses its TDMA schedule to transmit its data to its next hop. When a non- $\mathrm{CH}$ node (a $\mathrm{CM}$ or an $\mathrm{SCH}$ ) finishes its data transmission slot, it enters the sleep state to save its energy. Fig. 4 shows the schedule of set-up and steady-state phases in a given round, in PSO-HC.

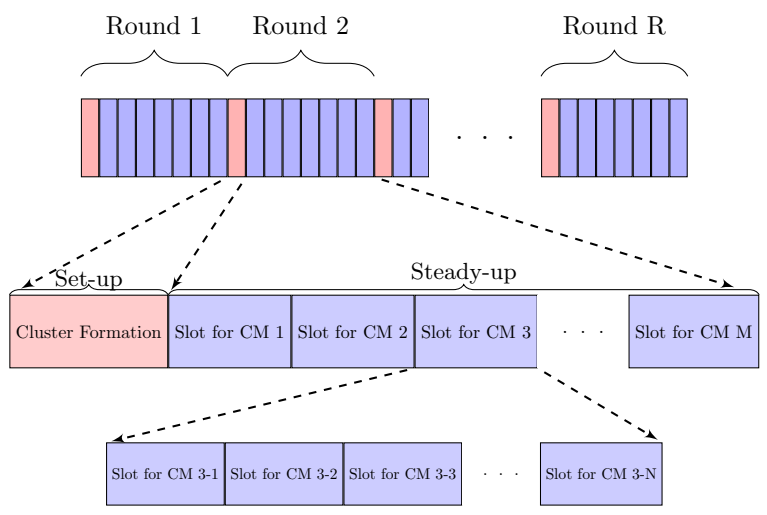

Fig. 4. Schedule of set-up and steady-state phases in a given round, in PSO-HC

\section{Simulations And Results}

In this section, the performance of PSO-HC is investigated against the well known protocols LEACH, LEACH-C and PSO-C. Simulations were carried on Castalia [23], which is based on the OMNeT++ platform and can be used to test WSN protocols in realistic wireless channel and radio models, with a realistic node behaviour.

The simulations were performed on 6 different network sizes ranging from 60 to 400 sensor nodes, and each network 
was tested using 5 different random seeds. The sensor nodes were deployed randomly in an area of $100 \mathrm{~m} \times 100 \mathrm{~m}$ sensor field. The protocol run for 5000 seconds (50 rounds). The upper bound on the number of $\mathrm{CHs}$ was set to $5 \%$ of the total nodes as used in [7], [8], [12]. The initial energy of a standard node is set to $E=18720$ Joules, which is the typical energy of two AA batteries [23]. We present the results at $99 \%$ confidence interval.

Table 1 exhibits the simulation settings summary of the simulation environment.

TABLE I

SIMULATION SETTINGS

\begin{tabular}{ll}
\hline Parameter & Value \\
\hline Initial energy & $18720 \mathrm{~J}$ \\
BS location & $(0,0)$ \\
Packets rate & 1 packet/s \\
Network size & $(60-400)$ sensor nodes \\
Field size & $100 m \times 100 m$ \\
MAC protocol & TMAC \\
Maximum number of CHs & $5 \%$ \\
Simulation time & $5000 \mathrm{~s}$ \\
Round length & $100 \mathrm{~s}$ \\
Slot length & $0.4 \mathrm{~s}$ \\
Number of particles & 50 \\
Number of iterations & 500 \\
Particles topology & Ring \\
\hline
\end{tabular}

\section{A. Energy Efficiency}

In Fig. 5, we investigate the energy efficiency of PSO-HC, compared to the other well known clustering protocols, by reporting the average energy consumption per node in joules when $w c_{1}=w c_{2}=1$. It is clearly shown that PSO-HC has lower energy consumption than the other protocols. This is a result of using less number of $\mathrm{CHs}$ as justified by using (3). Moreover, the results are shown to be statistically significant at $99 \%$ confidence interval.

Higher energy consumption was recorded in LEACH because the un-clustered nodes are left unattended without any sleeping schedule. Theoretically, both LEACH-C and PSO-C cluster all the network nodes and thus give each node a sleep schedule depending on its TDMA turn to transmit. This caused both protocols to have lower energy consumption compared to that of LEACH.

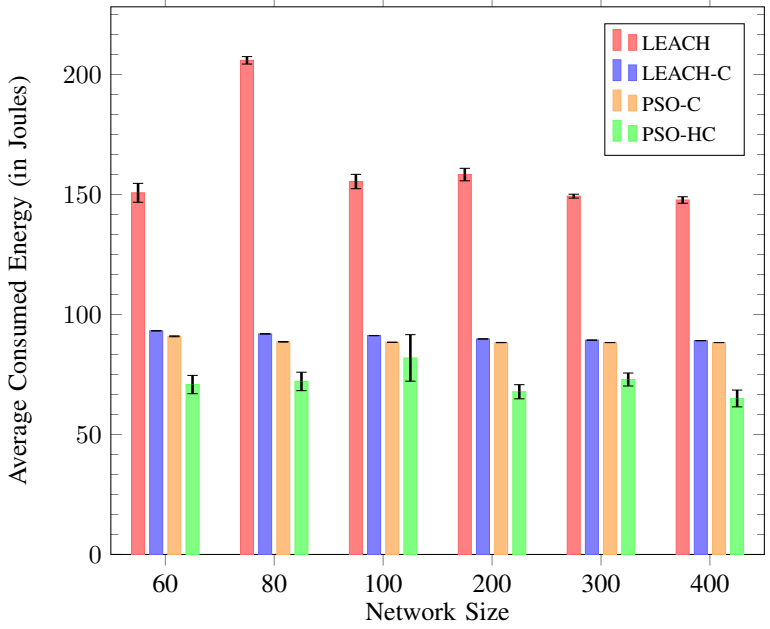

Fig. 5. Average Energy Consumption per Node

Fig. 6 examines the average energy consumption per node in joules in PSO-HC when $w c_{2}=1$ and for two different values of $w c_{1}, w c_{1}=0$ and $w c_{1}=1$. It is clearly shown that ignoring the energy efficiency objective, justified by (3) leads to higher energy consumption in most of the cases.

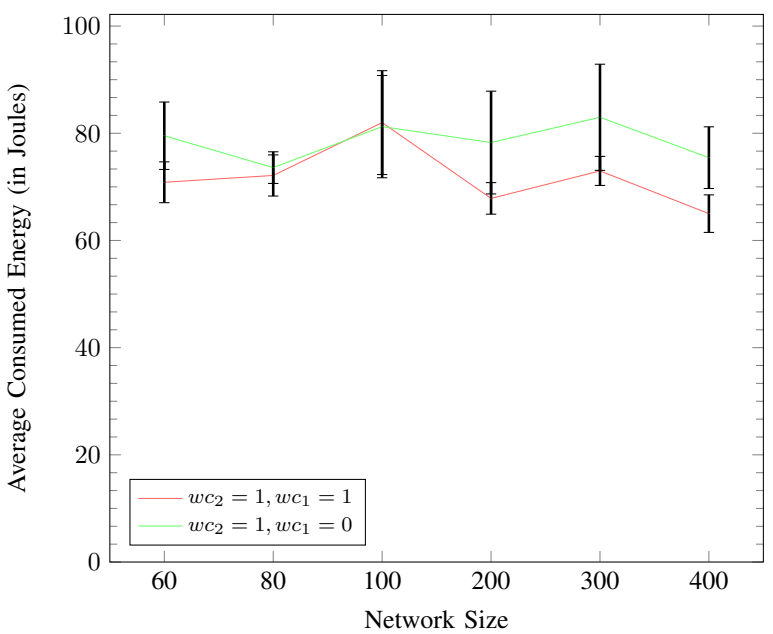

Fig. 6. Average Energy Consumption in PSO-HC for different values of $w c_{1}$

As in terms of throughput, Fig. 7 shows that the throughput, which is defined as the total number of data packets received by all the $\mathrm{CHs}$, is not affected much with the change in $w c_{1}$ value. 


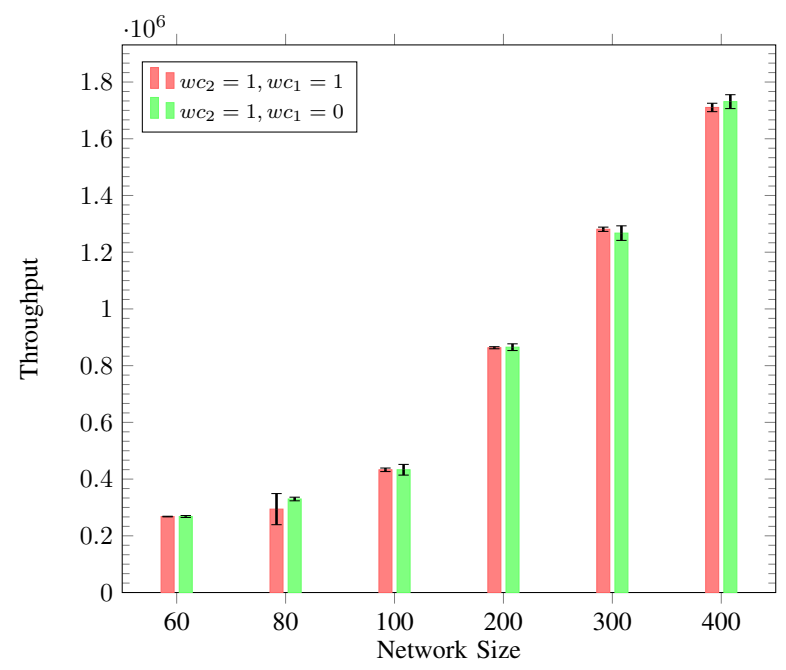

Fig. 7. Throughput in PSO-HC for different values of $w c_{1}$

\section{B. Link Quality}

In Fig. 8, we investigate the link quality in PSO-HC, compared to the other protocols, by reporting the throughput when $w c_{1}=w c_{2}=1$. It is clearly shown that PSO$\mathrm{HC}$ has higher throughput than the other protocols. This is a result of maximizing the link quality as justified by using (5). Moreover, results are shown to be statistically significant at $99 \%$ confidence interval.

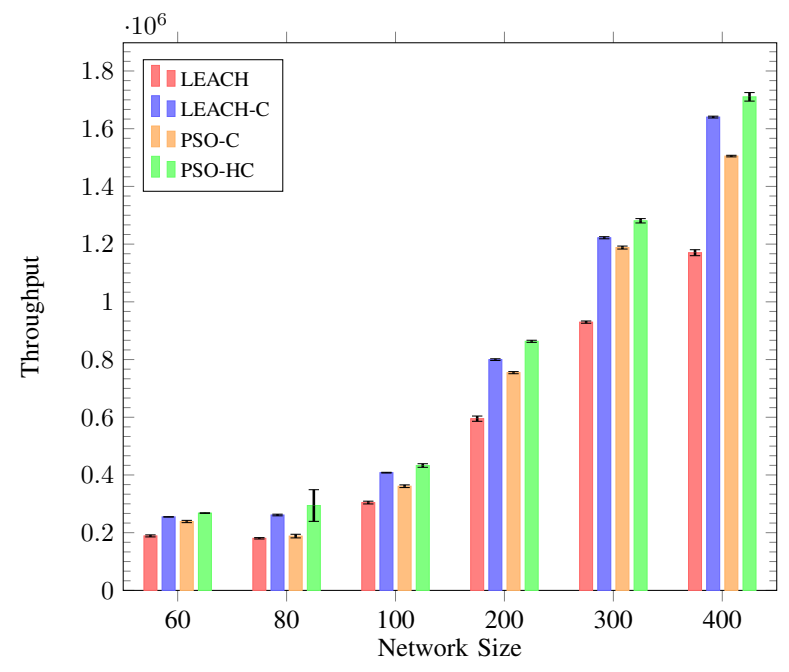

Fig. 8. Throughput

Fig. 9 examines the throughput in PSO-HC when $w c_{1}=1$ and for two different values of $w c_{2}, w c_{2}=0$ and $w c_{2}=1$. It is clearly shown that ignoring the link quality objective (5) leads to minimizing the throughput in PSO-HC.

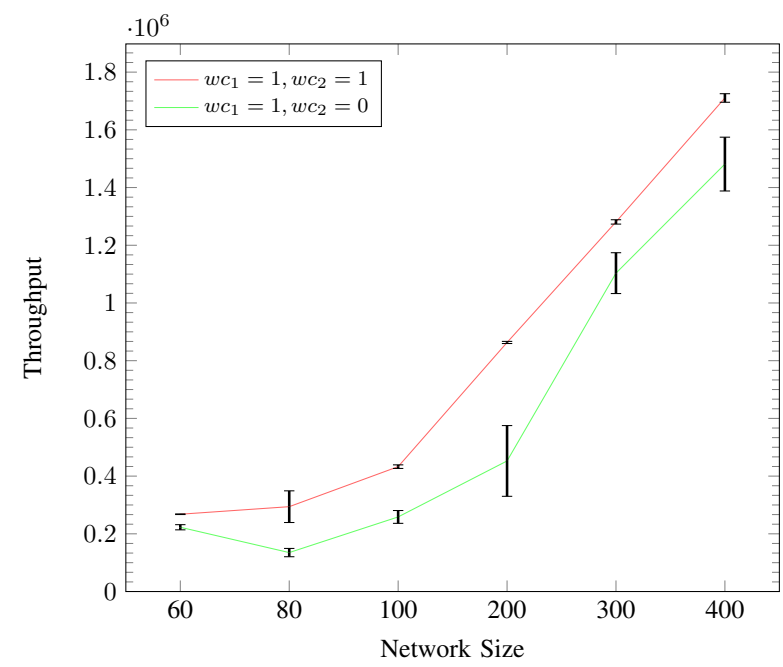

Fig. 9. Throughput PSO-HC for different values of $w c_{2}$

Fig. 10 shows that focusing only on minimizing the number of the primary $\mathrm{CHs}$ leads to lower energy consumption compared to taking into account the link quality objective also.

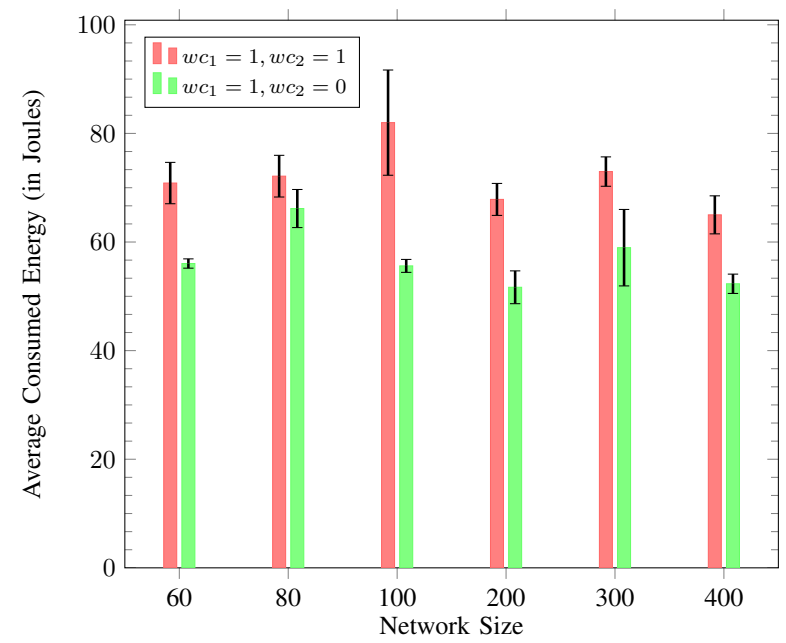

Fig. 10. Average Energy Consumption in PSO-HC for different values of $w c_{2}$

\section{CONCLUSION AND FUtURE WORK}

In this paper, a PSO-based hierarchical clustering protocol was developed for WSNs. The protocol enhances WSN energy efficiency by setting an upper bound on the number of $\mathrm{CHs}$ and trying to minimize the number of $\mathrm{CHs}$ compared to that upper bound. Furthermore, it enhances the network scalability by using two-tier cluster structure. The protocol was developed and tested under realistic network and energy consumption model. Extensive simulations were conducted, and the results show that the proposed protocol can improve the energy efficiency and throughput of WSN.

Future research directions can be inspired from the reported results. The protocol can be extended to allow N-tier hierarchical clusters with a threshold on the link quality between any $\mathrm{CM}$ and its next hop. This may result in better clusters quality 
and maximize the throughput. Furthermore, an inter-cluster communication method can be developed for transmitting the data packets to the BS.

\section{REFERENCES}

[1] A. K. Dwivedi and O. P. Vyas, "Article:network layer protocols for wireless sensor networks: Existing classifications and design challenges," International Journal of Computer Applications, vol. 8, no. 12, pp. 30-34, October 2010, published By Foundation of Computer Science.

[2] A. A. Abbasi and M. Younis, "A survey on clustering algorithms for wireless sensor networks," Computer Communications, vol. 30, no. 1415, pp. 2826 - 2841, 2007.

[3] P. K. Agarwal and C. M. Procopiuc, "Exact and approximation algorithms for clustering," Algorithmica, vol. 33, no. 2, pp. 201-226, 2002.

[4] R. Kulkarni and G. Venayagamoorthy, "Particle swarm optimization in wireless-sensor networks: A brief survey," IEEE Transactions on Systems, Man, and Cybernetics, Part C: Applications and Reviews, vol. 41, no. 2, pp. 262-267, Mar. 2011.

[5] Y. Del Valle, G. Venayagamoorthy, S. Mohagheghi, J.-C. Hernandez, and R. Harley, "Particle swarm optimization: Basic concepts, variants and applications in power systems," IEEE Transactions on Evolutionary Computation, vol. 12, no. 2, pp. 171-195, April 2008.

[6] O. Younis, M. Krunz, and S. Ramasubramanian, "Node clustering in wireless sensor networks: recent developments and deployment challenges," IEEE Network, vol. 20, no. 3, pp. 20-25, May 2006.

[7] W. Heinzelman, A. Chandrakasan, and H. Balakrishnan, "Energyefficient communication protocol for wireless microsensor networks," in Proceedings of the 33rd Annual Hawaii International Conference on System Sciences, vol. 2, Jan. 2000.

[8] _ - "An application-specific protocol architecture for wireless microsensor networks," IEEE Transactions on Wireless Communications, vol. 1, no. 4, pp. 660-670, Oct. 2002.

[9] L. M. Arboleda and N. Nasser, "Comparison of clustering algorithms and protocols for wireless sensor networks," in IEEE Canadian Conference on Electrical and Computer Engineering, May 2006, pp. 17871792.

[10] O. Younis and S. Fahmy, "HEED: a hybrid, energy-efficient, distributed clustering approach for ad hoc sensor networks," IEEE Transactions on Mobile Computing, vol. 3, no. 4, pp. 366-379, Oct. 2004.

[11] M. Ye, C. Li, G. Chen, and J. Wu, "Eecs: an energy efficient clustering scheme in wireless sensor networks," in Performance, Computing, and
[11] M. Ye, C. Li, G. Chen, and J. Wu, "Eecs: an energy efficient clustering scheme in wireless sensor networks," in Performance, Computing, and Communications Conference, 2005. IPCCC 2005. 24th IEEE International, April 2005, pp. 535-540.

[12] N. Latiff, C. Tsimenidis, and B. Sharif, "Energy-aware clustering for wireless sensor networks using particle swarm optimization," in IEEE 18th International Symposium on Personal, Indoor and Mobile Radio Communications (PIMR C'07), Sep. 2007, pp. 1-5.

[13] N. Abdul Latiff, C. Tsimenidis, and B. Sharif, "Performance comparison of optimization algorithms for clustering in wireless sensor networks," in IEEE Internatonal Conference on Mobile Adhoc and Sensor Systems. MASS 2007, Oct. 2007, pp. 1-4.

[14] C. jiang Jiang, W. ren Shi, min Xiang, and X. lun Tang, "Energybalanced unequal clustering protocol for wireless sensor networks," The Journal of China Universities of Posts and Telecommunications, vol. 17, no. 4, pp. $94-99,2010$.

[15] G. Molina and E. Alba, "Location discovery in wireless sensor networks using metaheuristics," Applied Soft Computing, vol. 11, no. 1, pp. 1223 - 1240, 2011.

[16] K. Srinivasan, P. Dutta, A. Tavakoli, and P. Levis, "An empirical study of low-power wireless," ACM Transactions on Sensor Networks, vol. 6, no. 2, pp. 16:1-16:49, Mar. 2010.

[17] N. Baccour, A. Koubâa, L. Mottola, M. A. Zúñiga, H. Youssef, C. A Boano, and M. Alves, "Radio link quality estimation in wireless sensor networks: A survey," ACM Transactions on Sensor Networks (TOSN), vol. 8, no. 4, pp. 34:1-34:33, Sep. 2012.

[18] K. Srinivasan and P. Levis, "RSSI is under appreciated," in Proceedings of the Third Workshop on Embedded Networked Sensors (EmNets 2006), May 2006.

[19] K. Srinivasan, P. Dutta, A. Tavakoli, and P. Levis, "Understanding the causes of packet delivery success and failure in dense wireless sensor networks," in Proceedings of the 4th International Conference on Embedded Networked Sensor Systems, Nov. 2006, pp. 419-420.

[20] "Chipcon CC2420 radio transceiver data sheet," http://inst.eecs.berkeley.edu/ cs150/Documents/CC2420.pdf, Last access: April 2, 2014.

[21] A. Barberis, L. Barboni, and M. Valle, "Evaluating energy consumption in wireless sensor networks applications," in 10th Euromicro Conference on Digital System Design Architectures, Methods and Tools, 2007. DSD 2007., Aug. 2007, pp. $455-462$.

[22] J. Kennedy and R. Eberhart, "Particle swarm optimization," in Proceedings of IEEE International Conference on Neural Networks, 1995., vol. 4, Nov./Dec. 1995, pp. $1942-1948$. 